\title{
Defining a physical basis for diversity in protein self-assemblies using a minimal model
}

Srivastav Ranganathan, Samir K Maji, Ranjith Padinhateeri 


\section{Supporting Figures}

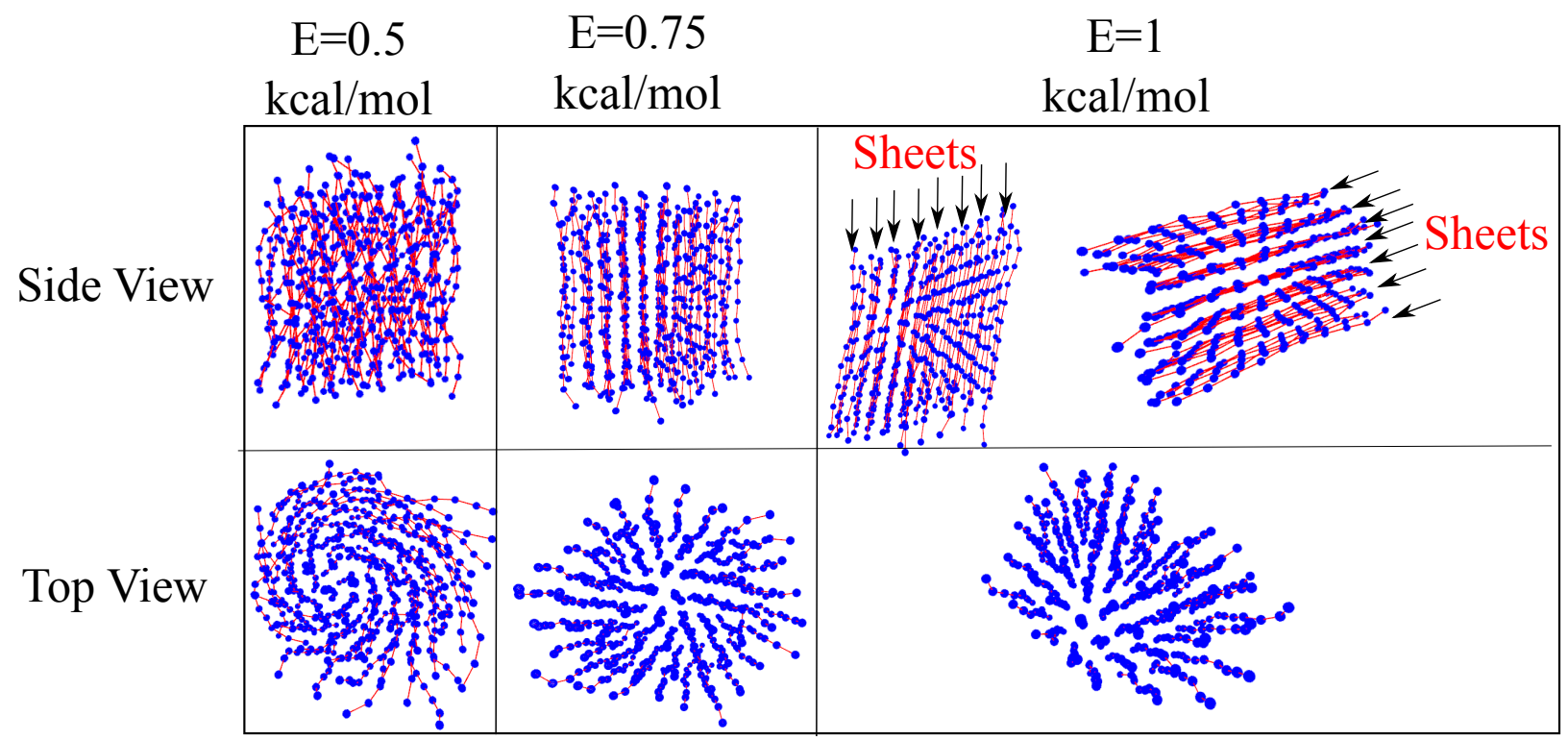

Figure S1. Different representations of the ordered aggregated structures formed by constituent chains of $\kappa=10 \mathrm{kcal} / \mathrm{mol}$, at various values of $\epsilon$. 


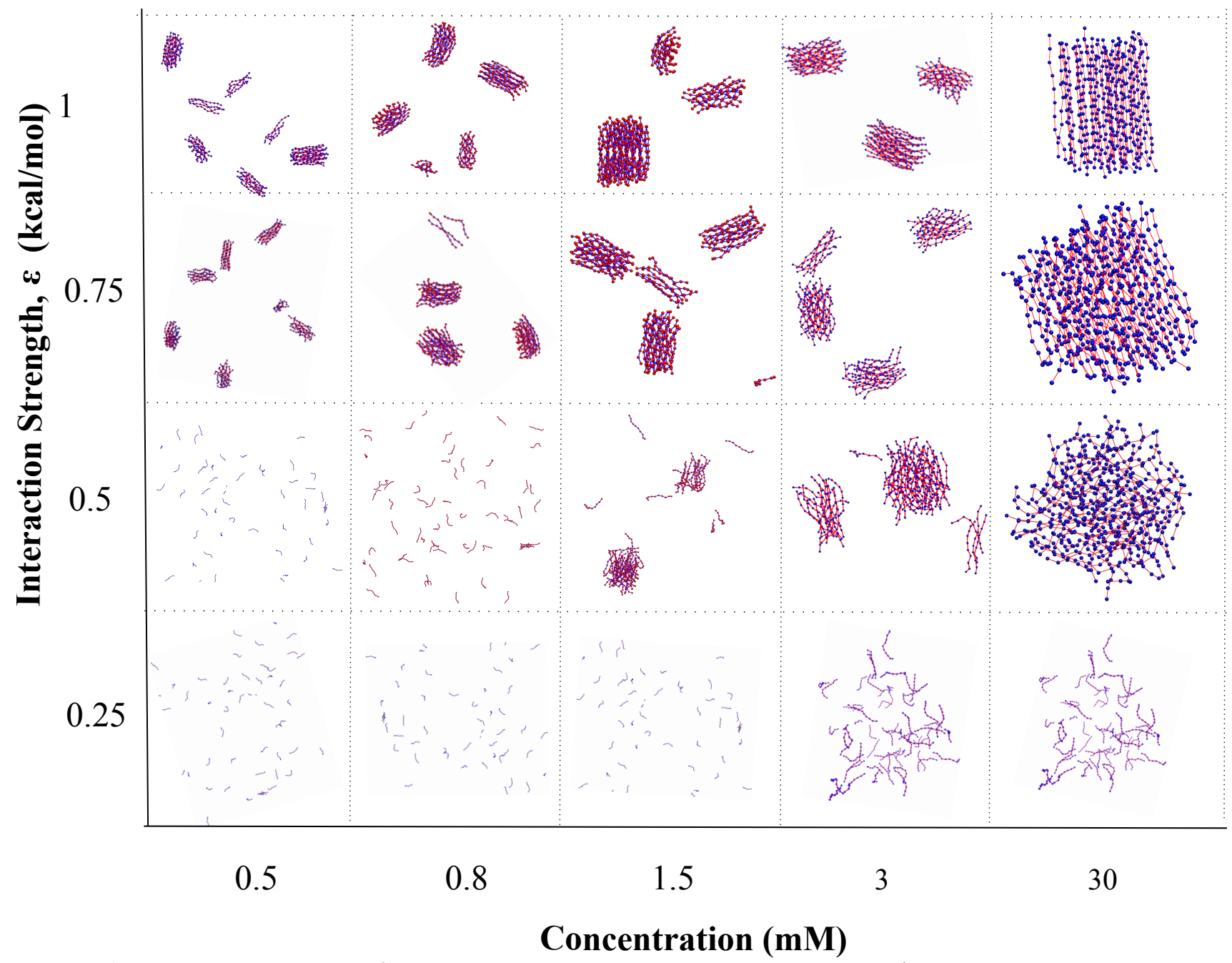

Figure S2. The aggregation profile in response to varying concentration and $\epsilon$. At low concentration and low $\epsilon$, the system is mostly monomeric. At low concentration and higher values of $\epsilon$, small aggregated clusters appear. At very high conentration, the system forms a single large aggregated cluster.
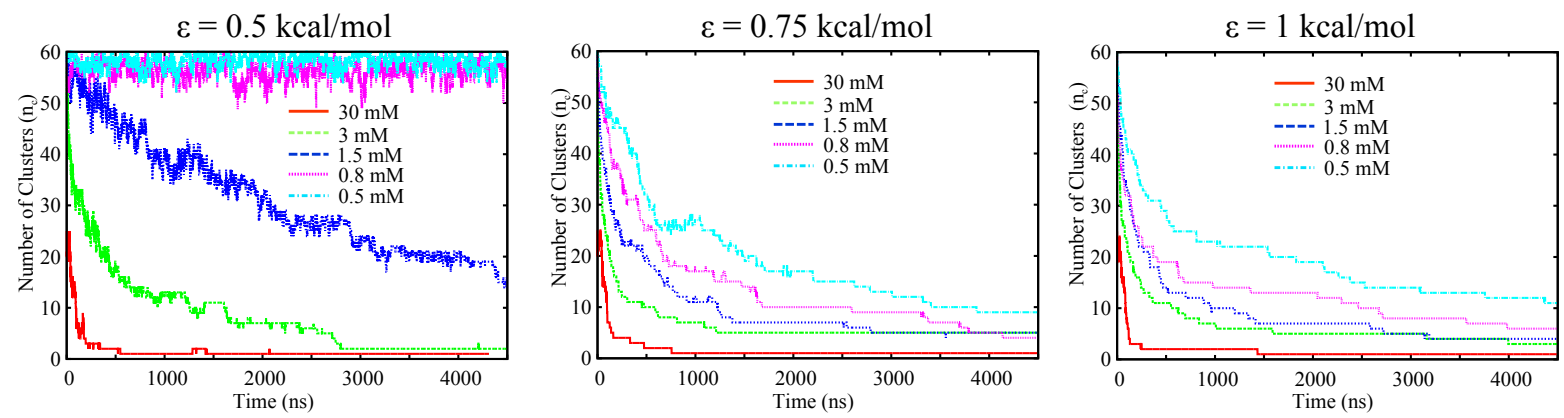

Figure S3. Cluster sizes $\left(n_{c}\right)$ as a function of time plotted for various values of concentration and $\epsilon$. A large $n_{c}$ refers to a predominanyl monomeric system while $n_{c} \rightarrow 1$ suggests a single aggregate of large size. 


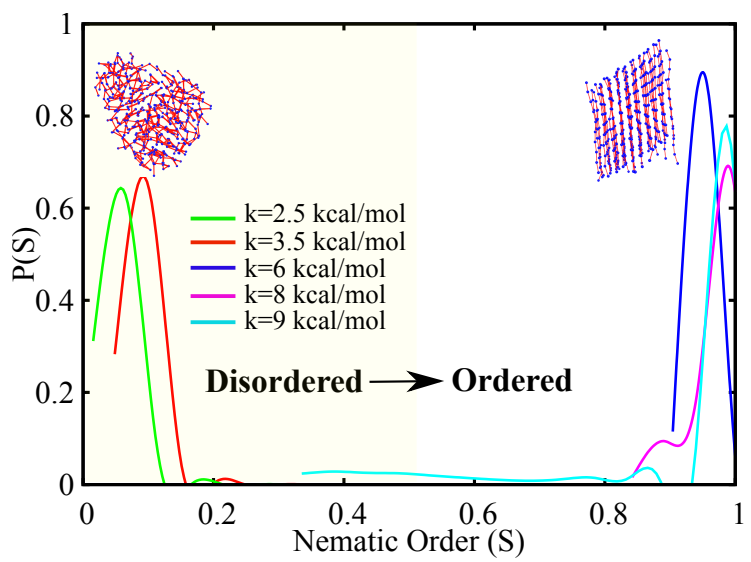

Figure S4. Probability distribution of the ordered states accessed by the polymer chains during self-assembly at an $\epsilon$ value of $1 \mathrm{kcal} / \mathrm{mol}$, for various values of $\kappa$.

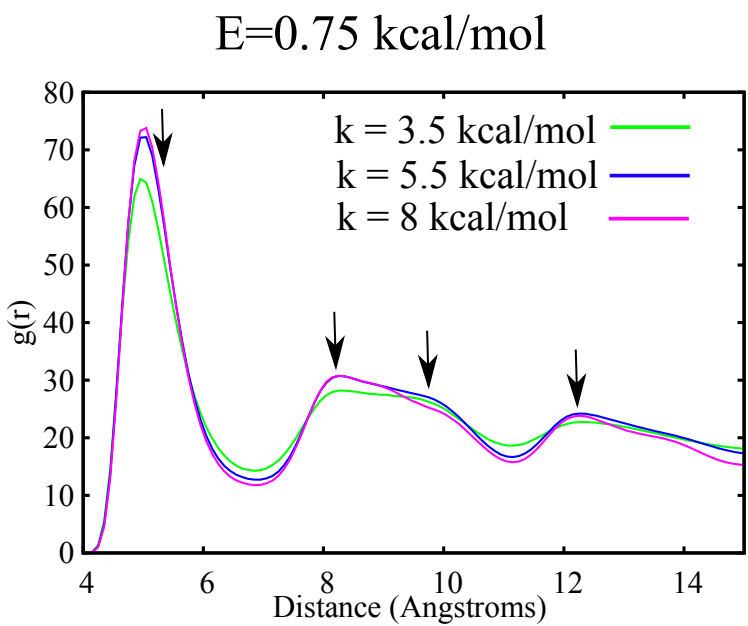

Figure S5. Radial distribution function for the aggregates formed by polymers of different bending stiffness, at an $\epsilon$ value of $0.75 \mathrm{kcal} / \mathrm{mol}$. 


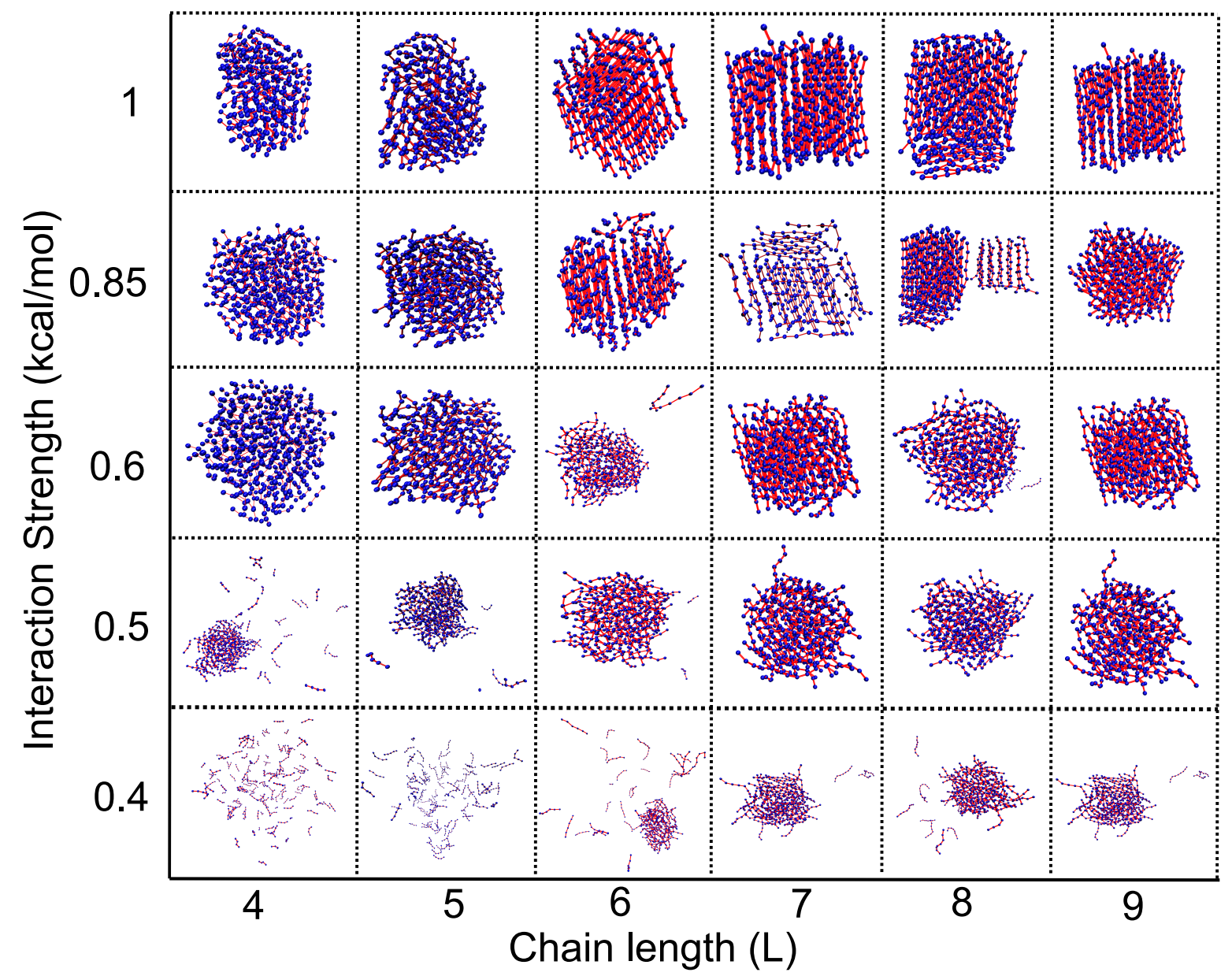

Figure S6. Aggregate morphology in response to varying constituent chain length (L) and interaction strengths. 

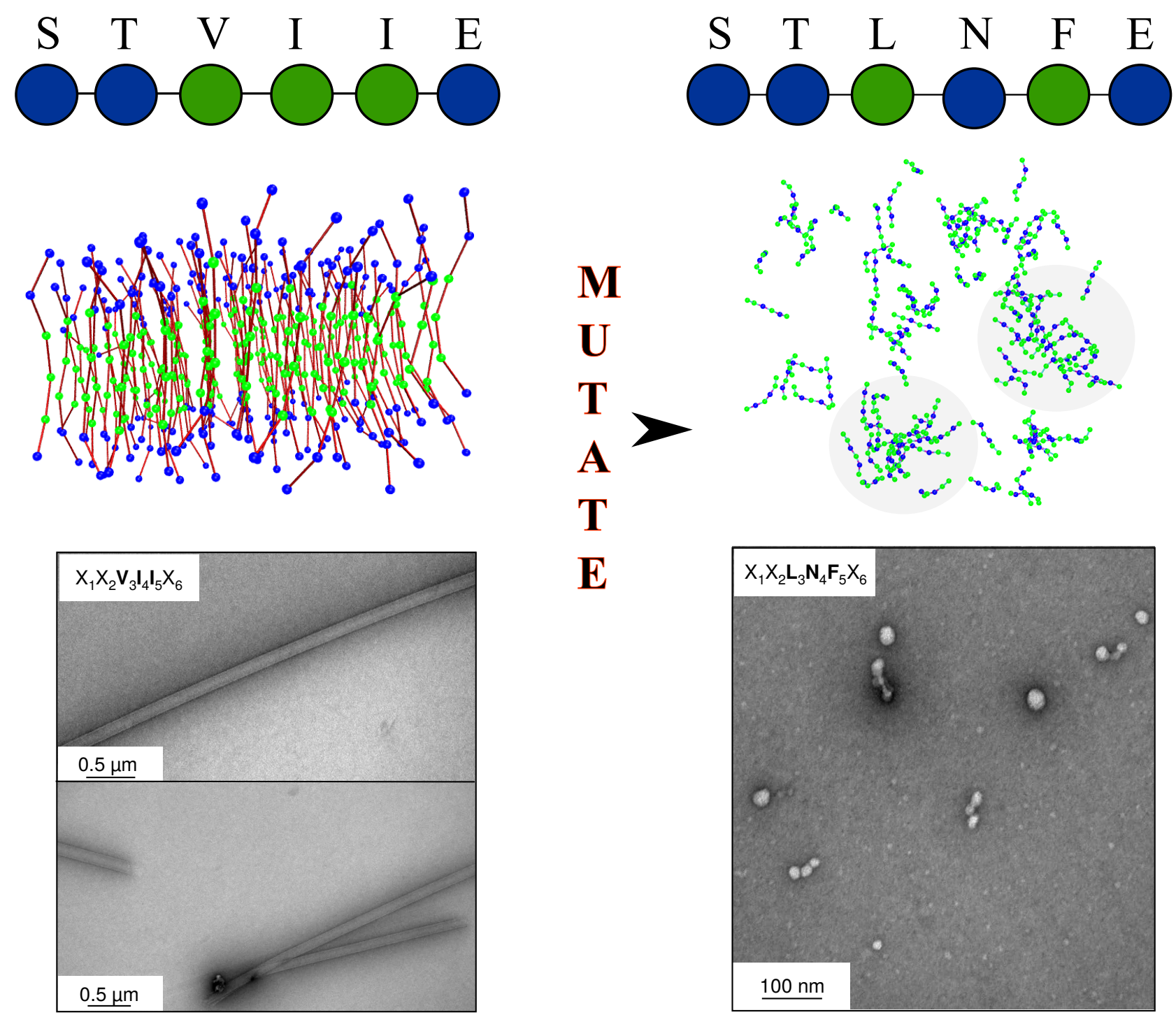

$\mathbf{E}$

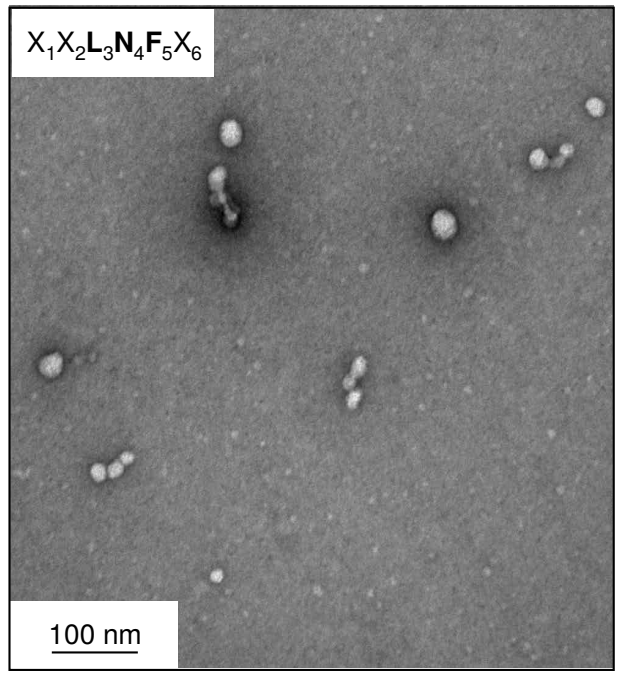

Figure S7. Effect of mutations to the 3rd, 4 th and 5 th positions of the STVIIE peptide. The EM images are taken from Lopez and Serrano [1]. 


\section{References}

[1] Manuela Lopez de la Paz and Luis Serrano. Sequence determinants of amyloid fibril formation. Proc. Natl. Acad. Sci. U. S. A., 101(1):87-92, 2004. 